\title{
Counterbalancing the Hydroxychloroquine Induced QT Prolongation: COVID 19 and Beyond
}

\author{
Ertan Yetkin ${ }^{1}$, Didem Tascioglu $^{2}$, Sirin Yurtlu Temel ${ }^{1}$, Bilal CUGLAN $^{3}$, and Kenan Yalta ${ }^{4}$ \\ ${ }^{1}$ Istinye University \\ ${ }^{2}$ Istinye Universitesi \\ ${ }^{3}$ Beykent University \\ ${ }^{4}$ Trakya Universitesi
}

August 6, 2020

\begin{abstract}
Those patients with progressive QT prolongation or QT interval exceeding $>500 \mathrm{~ms}$ may complete the COVID 19 treatment protocol containing HCQ and/or AZM without serious arrhythmias and torsades de pointes by the adding the mexiletine or lidocaine as an adjunctive drug in those patients. Counterbalancing the QT prolongation by mexiletine in association with would further decrease the labor and stress of healthcare providers during the continuing COVID 19 pandemic. Although it is a big dilemma how the therapeutic or prophylactic use of HCQ will end up, the pathophysiologic effects of HCQ on QT interval and counterbalancing by antiarryhthmic drugs mexiletine and lidocaine is out of the current discussion of COVID 19 pandemics. Counter balancing effect of mexiletine and lidocaine on QT interval in patients receiving HCQ would be helpful in patients with not only COVID 19 but also in malaria and rheumatologic disease as well.
\end{abstract}

Counterbalancing the Hydroxychloroquine Induced QT Prolongation: COVID 19 and Beyond

Ertan Yetkin ${ }^{1}$ MD

Didem Tascioglu² MD

Sirin Yurtlu Temel ${ }^{3}$ MD

Bilal Cuglan ${ }^{4} \mathrm{MD}$

Kenan Yalta ${ }^{5}$ MD

${ }^{1}$ Liv Hospital, Department of Cardiology Istanbul Turkey

${ }^{2}$ Liv Hospital Department of Infectious Disease, Istanbul Turkey

${ }^{3}$ Liv Hospital Department of Pulmonary Disease, Istanbul Turkey

${ }^{4}$ Beykent University, Faculty of Medicine Istanbul Turkey

5 Trakya University Faculty of Medicine Department of Cardiology, Edirne Turkey

Address for correspondence: Ertan Yetkin

Istinye University Faculty of Medicine Department of Cardiology Liv Hospital, Bahcesehir 34880, Istanbul Turkey

Tel: 00904446623 
Fax: 02129794000

Email: ertanyetkin@hotmail.com

Counterbalancing the Hydroxychloroquine Induced QT Prolongation: COVID 19 and Beyond

Abstract

Those patients with progressive QT prolongation or QT interval exceeding $>500$ ms may complete the COVID 19 treatment protocol containing HCQ and/or AZM without serious arrhythmias and torsades de pointes by the adding the mexiletine or lidocaine as an adjunctive drug in those patients. Counterbalancing the QT prolongation by mexiletine in association with would further decrease the labor and stress of healthcare providers during the continuing COVID 19 pandemic. Although it is a big dilemma how the therapeutic or prophylactic use of HCQ will end up, the pathophysiologic effects of HCQ on QT interval and counterbalancing by antiarryhthmic drugs mexiletine and lidocaine is out of the current discussion of COVID 19 pandemics. Counter balancing effect of mexiletine and lidocaine on QT interval in patients receiving HCQ would be helpful in patients with not only COVID 19 but also in malaria and rheumatologic disease as well.

Key words: QT prolongation, lidocaine, mexiletine, COVID 19, Hydroxychloroquine

The rapid spreading severe acute respiratory syndrome coronavirus has led to an ongoing pandemic of coronavirus disease 2019 (COVID 19) with its own transmissibility pattern and mortality features. Beyond the direct involvement of cardiovascular system and contribution of hypertension and cardiac disease on the clinical courses of patients with COVID 19, drug induced QT prolongation and severe ventricular arrhythmia has emerged as a new worrisome issue by the widespread use of hydroxychloroqin and/or azithromycin (AZM) treatment against COVID 19. In this regard Mercuroet al' report underlines risk of QT prolongation in hospitalized patients receiving $\mathrm{HCQ}$ and $\mathrm{AZM}^{1}$. Nearly one out of 5 patients have been reported to have either QTc prolongation >500 ms or increase in QTc interval >60 ms. Thereby they have highlighted the importance of weighing the risks and benefits when considering hydroxychloroquine and azithromycin, with close monitoring of QTc and concomitant medication usage. Recently Chorin et $\mathrm{al}^{2}$ has reported QT prolongation $>40 \mathrm{~ms}$ in 30\% of COVID 19 patients treated with HCQ and AZM combination. Moreover $11 \%$ of those patients have reached critical level of QTc prolongation $>500 \mathrm{~ms}$ representing high risk group of arrhythmia. Likewise, Chang et $\mathrm{al}^{3}$ has reported $34 \mathrm{~ms}$ increase in QT interval for the entire COVID19 patients and discontinuation of HCQ due to progressive QT prolongation from $460 \mathrm{~ms}$ to $565 \mathrm{~ms}$ in one patient by a novel outpatient monitoring system. Given the fact that $\mathrm{HCQ} \pm \mathrm{AZM}$ may increase the risk for arrhythmias and sudden cardiac death due to QT prolongation, it might also be a reasonable approach to shorten the prolonged or progressively prolonging QT interval in patients under the treatment of HCQ and AZM thereby reducing the risk of severe arrhythmias. Quinoline derivatives such as natural quinine, quinidine, and synthetically produced HCQ have been used for treatment of malaria, arrhythmias and rheumatic diseases for decades. Of note, quinidine has remained a historical Class I-a anti-arrhythmic drug which acts through the inhibition of fast $\mathrm{Na}$ channel with depression of phase $\mathrm{O}$ action potential and to lesser extend blocking outward potassium current thereby prolonging QT interval. Class I-b anti-arrhythmic drugs namely, lidocaine and mexiletine also inhibit the fast $\mathrm{Na}$ channel especially in non-diseased myocardium which results in QT shortening rather than prolongation. Indeed mexiletine has been used in treatment of severe ventricular arrhythmia in combination with quinidine to achieve high success rate and counterbalance the pro-arrhythmic effects of quinidine. Recently Bos et $\mathrm{al}^{4}$ has shown that the sodium channel blocker mexiletine has produced a substantial, and clinically relevant, shortening of the QT interval among patients with long QT syndromes. In addition to long QT syndrome mexiletine has reduced not only the QT interval but also the recurrence of torsades de pointes in patients with acquired long QT secondary to dofetilide, and amiodaron ${ }^{5}$. Mexiletine-quinidine combination therapy has resulted in higher anti-arrhythmic success rate and lower adverse effects compared with the quinidine monotherapy ${ }^{6}$. Moreover quinidine-mexiletine combination therapy has not led an increase in QT interval, in contrast to quinidine monotherapy compared to baseline values ${ }^{7}$. Intravenous lidocaine has significantly shortened QT interval from $620 \mathrm{~ms}$ to $550 \mathrm{~ms}$ thereby allowing administration of HCQ and AZM in a critically ill patients with COVID $19^{8}$. 
Regarding the salutary and old interaction of mexiletine and quinidine which has ended up with higher anti-arrhythmic effect, lower side effect and absence of QT prolongation, we may propose adjunctive use of mexiletine with HCQ in patients with COVID- 19 to overwhelm the risk of QT prolongation and severe arrhythmia. Those patients with progressive QT prolongation or QT interval exceeding $>500$ ms may complete the COVID 19 treatment protocol containing HCQ and/or AZM without serious arrhythmias and torsades de pointes by the adding the mexiletine or lidocaine as an adjunctive drug in those patients. Counterbalancing the QT prolongation by mexiletine in association with would further decrease the labor and stress of healthcare providers during the continuing COVID 19 pandemic.

Based on the current emerging scientific data FDA has withdrawn the emergency use authorization (EUA) to use hydroxychloroquine to treat COVID-19 in certain hospitalized patients. Although it is a big dilemma how the therapeutic or prophylactic use of HCQ will end up, the pathophysiologic effects of HCQ on QT interval and counterbalancing by antiarryhthmic drugs mexiletine and lidocaine is out of the current discussion of COVID 19 pandemics. Counter balancing effect of mexiletine and lidocaine on QT interval in patients receiving HCQ would be helpful in patients with not only COVID 19 but also in malaria and rheumatologic disease as well.

References:

Mercuro JN, Yen CF,Shim DJ, MD, et alRisk of QT Interval Prolongation Associated With Use of Hydroxychloroquine With or Without Concomitant Azithromycin Among Hospitalized Patients Testing Positive for Coronavirus Disease 2019 (COVID-19) JAMA Cardiol. Published online May 1, 2020. doi:10.1001/jamacardio.2020.1834

1. Chorin, E., Dai, M., Shulman, E. et al. The QT interval in patients with COVID-19 treated with hydroxychloroquine and azithromycin. Nat Med (2020). https://doi.org/10.1038/s41591-020-0888-2

2. Chang D, Saleh M, Gabriels J, Ismail H, Goldner B, Willner J, Beldner S, Mitra R, John R, Epstein LM. Inpatient Use of Ambulatory Telemetry Monitors for COVID-19 Patients Treated with Hydroxychloroquine and/or Azithromycin. J Am Coll Cardiol. 2020 Apr 18. pii: S0735-1097(20)35009-9. doi: 10.1016/j.jacc.2020.04.032. [Epub ahead of print]

3. Bos JM, Crotti L, Rohatgi RK, Castelletti S, Dagradi F, Schwartz PJ, Ackerman MJ. Mexiletine Shortens the QT Interval in Patients with Potassium Channel-Mediated Type2 Long QT Syndrome. Circ Arrhythm Electrophysiol. 2019;12(5):e007280. doi: 10.1161/CIRCEP.118.007280.

4. Badri M, Patel A, Patel C, Liu G, Goldstein M, Robinson VM, Xue X, Yang L, Kowey PR, Yan GX. Mexiletine prevents recurrent torsades de pointes in acquired long qt syndrome refractory to conventional measures. JACC Clin Electrophysiol. 2015;1:315-322. doi: 10.1016/j.jacep.2015.05.008

5. Giardina EG, Wechsler ME. Low dose quinidine-mexiletine combination therapy versus quinidine monotherapy for treatment of ventricular arrhythmias. J Am Coll Cardiol. 1990;15(5):1138-45.

6. Duff HJ, Mitchell LB, Manyari D, Wyse DG. Mexiletine quinidine combination: electrophysiologic correlates of favorable antiarrhythmic interaction in humans. J Am Coll Cardiol. 1987;10(5):1149-56.

7. Mitra RL, Greenstein SA, Epstein LM. An algorithm for managing QT prolongation in Coronavirus Disease 2019 (COVID-19) patients treated with either chloroquine or hydroxychloroquine in conjunction with azithromycin: Possible benefits of intravenous lidocaine. HeartRhythm Case Reports. 2020. 